\title{
An Experimental Study on the Effect of Adsorbing and Non-Adsorbing Block Sizes on Diblock Copolymer Adsorption
}

\author{
D. T. Wu, ${ }^{*}$ A. Yokoyama, and R. L. Setterquist \\ Marshall R\&D Laboratory, E. I. DuPont De Nemours \& Co., \\ P:O. Box 3886, Philadelphia, Pennsylvania 19146, U.S.A.
}

(Received November 26, 1990)

\begin{abstract}
To design diblock copolymers with optimum performance as stabilizers for colloidal dispersions, it is important to understand the relationships between surface coverage, layer thickness and block sizes. This work is an experimental study to gain this understanding using low polydispersity diblock copolymer of dimethyl amino ethyl methacrylate (DMAEM) and n-butyl methacrylate, adsorbed on narrow size distribution silica particles in 2-propanol. Both blocks are soluble in 2-propanol, whereas only DMAEM block adsorbs on silica in 2-propanol. With copolymers of constant degrees of polymerization of ca. 200 and ca. 700 , our experimental results show that very steep maximums both in adsorbed amount and in hydrodynamic layer thickness exist. The existence of these maximums was first predicted by the self-consistent field theory using a lattice model. For the adsorption regime dominated by packing of the non-adsorbing block, the hydrodynamic layer thickness was found to scale on the order of 0.53 , which is less that that of previous work.

KEY WORDS Adsorption / Diblock Copolymer / Silica / Poly (n-butyl methacrylate) / Poly (dimethyl amino ethyl methacrylate) / 2-Propanol / Hydrodynamic Layer Thickness / Photon Correlation Spectroscopy
\end{abstract}

Amphiphilic diblock copolymers are frequently used as stabilizers for colloidal dispersions, since the functions of the polymers for adsorption and stabilization can be divided between the two blocks. To design diblock copolymers with optimum performance as dispersion stabilizers, it is important to understand the relationships between surface coverage, layer thickness and block sizes. Evers, et al. ${ }^{1,2,3}$ have recently developed an adsorption theory for diblock copolymers based on the self-consistent field model. This theoretical model allows the prediction of surface coverage and hydrodynamic layer thickness as functions of several system parameters, such as the surface-polymer adsorption energies, the polymer-solvent interaction parameters, the degrees of polymerization and the compositions of the blocks. One of the significant findings of Evers' work is that both the surface coverage and the hydrodynamic layer thickness exhibit maximums as the composition of the adsorbing block is varied with a constant total degree of polymerization. This paper presents the results of our experimental work that can be compared with Evers' theoretical calculations. In addition, a number of other authors 4,5 have also developed theories for scaling of layer thicknesses for adsorbed diblock copolymers, and we compared the order of scaling of our results with theirs.

In our work, we used narrow size distribution silica spheres as model colloids and low polydispersity diblock copolymers synthesized by the group transfer living polymerization technique. One of the advantages of using the silica particles is that it is non-swellable in the presence of a variety of organic solvents. The adsorbing block is poly 
(dimethyl amino ethyl methacrylate) [DMAEM] whereas the non-adsorbing block is poly (n-butyl methacrylate) [BMA]. The solvent used was 2-propanol, and it was selected for its solubility characteristics and displacer strength for BMA block. Both blocks are soluble in 2-propanol: thus, concentration of the diblock copolymer can be varied without concerns for micellization. In the presence of 2-propanol, only DMAEM block adsorbs with BMA only functioning as a stabilizer. Isopropanol was also chosen for its high dielectric constant which allows electrostatic stabilization during adsorption, to minimize particles aggregation, and also allows the adsorption step to be carried out without involving the "dry" stage for the particles. By minimizing aggregation of particles, the hydrodynamic thickness could be accurately measured by photon correlation spectroscopy.

\section{EXPERIMENTAL}

Silica particles were prepared essentially by the method of Stoeber, et al. ${ }^{6}$, which involves hydrolysis of tetra ethyl ortho silicate in methanol containing varying amounts of ammonia and water. We have measured the polydispersity of the particles using both TEM and sedimentation field flow fractionation techniques. Polydispersity, expressed as the ratio of the weight average and number average particle diameter, was found to be 1.05 for a typical dispersion by counting 494 particles of a TEM picture. A typical TEM picture is shown in Figure 1. Using the sedimentation field flow fractionation technique, polydispersity was found to be 1.06. The silica particles used had a mean diameter of 200 nm.

The diblock copolymer of DMAEM and BMA was synthesized by the group transfer living polymerization process $^{7}$ to obtain copolymers with a narrow molecular weight distribution. First, BMA was polymerized in tetrahydrofuran using diethyl ketene methyl trimethylsilylacetal as the initiator and tetra butyl ammonium m-chloro benzoate as the catalyst at about $45^{\circ} \mathrm{C}$. After the desired B-block lengths of BMA had been obtained, DMAEM was added to grow A-block. Then, polymerization was terminated with methanol. The molecular weight and the composition were measured by gel permeation chromatography and amine titration, respectively.

A typical gel permeation chromatogram of the diblock copolymer is given in Figure 2. The chromatogram is not symmetrical, and there appears to be a "shoulder" for low molecular weight polymer fractions. "Living" polymerization has tendency to terminate prematurely, and it is believed that the low molecular fractions of our polymers which contribute to the polydispersity are mostly homopolymers of BMA. This reasoning is supported by comparing the GPC curves for the original diblock copolymer with that of the adsorbed copolymer after it has been removed using dimethyl ethanol amine as a displacer. This is shown as a dotted curve in Figure 2, and the properties of original and removed copolymers are listed in Table I. These results support the fact that the adsorbed copolymers were of low polydispersity and had a greater ratio of DMAEM. Thus, for

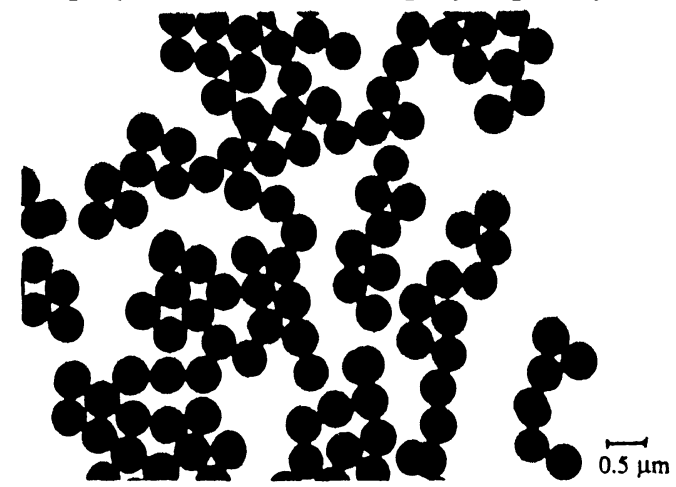

Figure 1. Transmission electron micrograph of a typical silica dispersion.

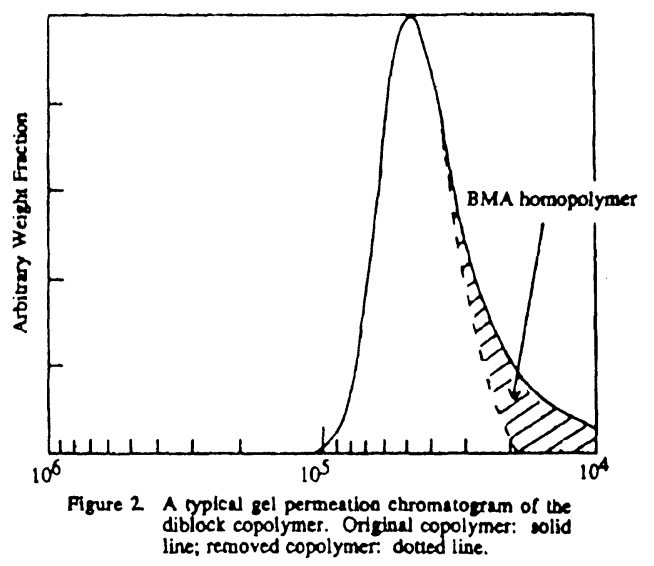

Polym. J., Vol. 23, No. 5, 1991 
polymers, of which we did not know the molecular weight and the block ratio of adsorbed copolymers, corrections were made in the average molecular weights and in the DMAEM mole percent, by assuming that the diblock copolymer fractions are represented by the symmetrical fractions of the GPC curve.

The adsorption process was carried out by mixing the silica particles with the polymer solution for 24 hours at $25^{\circ} \mathrm{C}$. The amount of adsorption was measured from the depleted amount of polymer in the supernatant. This amount of adsorption was reconfirmed by thermal gravimetric analysis of silica with the adsorbed copolymer. Typical results are shown in Table II.

The hydrodynamic layer thickness of the adsorbed polymer layer was determined by photon correlation spectroscopy (the cummulants method) ${ }^{8,9}$, which measured the hydrodynamic diameter of the particles. The difference in the diameters between the bare particles and the coated particles gives twice the hydrodynamic layer thickness. The scattering angle was varied from 30 - 150 degrees, and we observed a small decrease $(5 \%)$ in the diameter with an increase in the scattering angles from 30 - 150 degrees, indicating that both the bare particles and the coated particles were slightly polydisperse. However, the hydrodynamic layer thickness measured at varied scattering angles generally agreed within $2-3 \mathrm{~nm}$. Thus, the small polydispersity and whatever changes in polydispersity taking place during adsorption have no significant effect on our hydrodynamic layer thickness measurements. A typical result is shown in Figure 3, in which $\mathrm{Dq}^{2}$ is plotted against $\mathrm{q}^{2}$ (D: diffusion coefficient, q: scattering vector). The difference between the measured base-line and the calculated base-line was within a few tenths of a percent, indicating that the effect of dust is insignificant. In addition, the particle concentration was kept low enough to eliminate the inter-particle interactions.

Table I. Properties of polymers

\begin{tabular}{|c|c|c|c|c|c|c|c|c|c|}
\hline \multicolumn{5}{|c|}{ ORIGINAL POLYMER } & \multicolumn{5}{|c|}{$\begin{array}{l}\text { POLYMER REMOVED FROM SILICA SURFACE } \\
\text { (ADSORBED POLYMER) }\end{array}$} \\
\hline & $\begin{array}{l}\text { DMAEM } \\
\text { MOL } \%\end{array}$ & ${ }_{6} \mathrm{Mw}$ & $\mathrm{Mn}$ & $\mathrm{Mw} / \mathrm{Mn}$ & & $\begin{array}{l}\text { AEM } \\
\text { OL \% }\end{array}$ & Mw & $\mathrm{Mn}$ & $\mathrm{Mw} / \mathrm{Mn}$ \\
\hline$B_{142}$ & 0.0 & 22600 & 20200 & 1.11 & \multicolumn{5}{|c|}{ (NO ADSORPTION) } \\
\hline B429 & 0.0 & 75300 & 60900 & 1.24 & \multicolumn{5}{|c|}{ (NO ADSORPTION) } \\
\hline A5 B477 & 1.0 & 89700 & 68500 & 1.31 & $\mathrm{~A}_{8} \mathrm{~B} 610$ & 1.3 & 101100 & 87800 & 1.15 \\
\hline$A_{12} B_{468}$ & 2.5 & 92900 & 68400 & 1.36 & $A_{18}$ B769 & 2.3 & 128500 & 112000 & 1.15 \\
\hline $\mathrm{A}_{16} \mathrm{~B} 425$ & 3.6 & 105600 & 62900 & 1.68 & A27 B 665 & 3.9 & 131200 & 98700 & 1.33 \\
\hline $\mathrm{A} 36 \mathrm{~B} 420$ & 7.9 & 95700 & 65400 & 1.46 & $\mathrm{~A}_{109}$ B 649 & 14.4 & 131500 & 109400 & 1.20 \\
\hline$A_{174}$ & 100 & 39100 & 27500 & 1.42 & $A_{176}$ & 100 & 31900 & 27800 & 1.15 \\
\hline$A_{4} B_{141}$ & 2.8 & 27700 & 20700 & 1.34 & A7 B 197 & $3.4^{\mathrm{a}}$ & & $29000^{\mathrm{a}}$ & \\
\hline A8 B208 & 3.7 & 36700 & 30800 & 1.19 & $A_{11} B_{227}$ & $4.7^{\mathrm{a}}$ & & $34000^{a}$ & \\
\hline A42 B519 & 7.5 & 105400 & 80300 & 1.31 & A73 B705 & $9.4^{\mathrm{a}}$ & & $111600^{\mathrm{a}}$ & \\
\hline$A_{16} B_{176}$ & 8.3 & 27900 & 27500 & 1.01 & $\mathrm{~A}_{26} \mathrm{~B}_{183}$ & $12.4^{\mathrm{a}}$ & & $30000^{a}$ & \\
\hline
\end{tabular}

a estimated from the GPC curve of the original polymer

Table II Comparison between the amount of adsorption measured by TGA and that measured by the depletion method (A6 B430)

\begin{tabular}{ccc}
\hline $\begin{array}{c}\text { Equilibrium Concentration } \\
\text { [ppm] }\end{array}$ & $\begin{array}{c}\text { Thermal Gravimetry } \\
{\left[\mathrm{mg} / \mathrm{m}^{2}\right]}\end{array}$ & $\begin{array}{c}\text { Depletion Method } \\
{\left[\mathrm{mg} / \mathrm{m}^{2}\right]}\end{array}$ \\
\hline 500 & 2.4 & 2.4 \\
1000 & 3.3 & 3.5 \\
2000 & 4.5 & 5.2 \\
\hline
\end{tabular}




\section{RESULTS AND DISCUSSIONS}

In Figures 4 and 5, the amount of adsorption (plateau value) and the hydrodynamic layer thickness are plotted as a function of the composition in mole percent of the adsorbing block. Definitely both the adsorbed amount and the hydrodynamic layer thickness exhibit steep maximums as the adsorbing block composition increases, in agreement with the predictions of the theoretical model of Evers, et al. With no adsorbing block, the BMA block does not adsorb on silica. As the DMAEM block length increases, the adsorption energy increases due to increased interactions between the acidic silica surface and the basic DMAEM via acid-base interactions. This increase in the adsorption energy compensates the repulsion between the BMA blocks and leads to a greater amount of adsorption. With this increase in the amount of adsorption, the crowding effect between the BMA blocks becomes significant, leading to the stretched conformation of the BMA block (the greater hydrodynamic layer thickness).

However, as the DMAEM block length increases further, one diblock copolymer molecule occupies a larger area on the silica surface, owing to the flat conformation adopted by the DMAEM block. This increased area per molecule

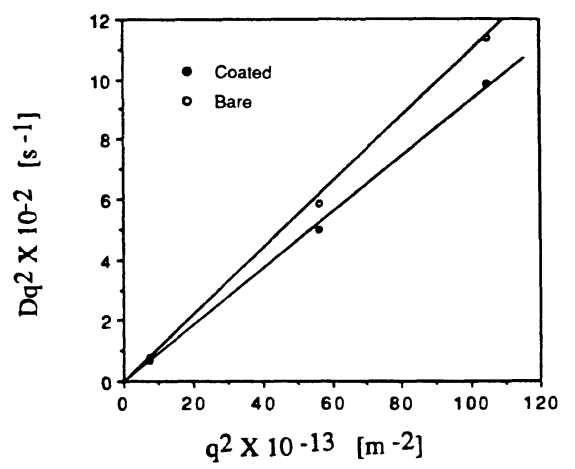

Figure 3. $\mathrm{Dq}^{2}$ against $\mathrm{q}^{2}$ over a scattering angle range from 30 to 150 o. A146 B377 (Mw/Mn: 1.15); hydrodynamic layer thickness: $17 \mathrm{~nm}$
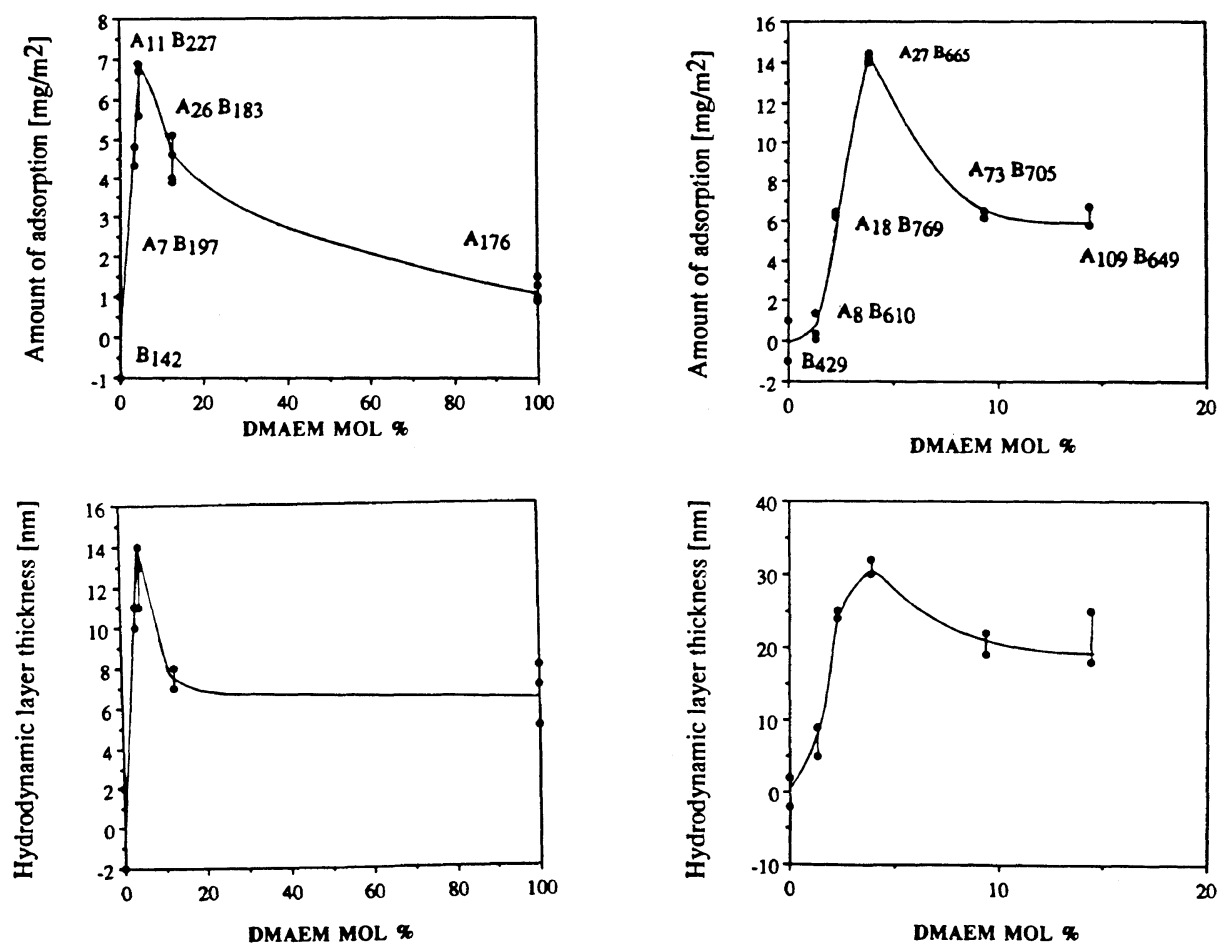

Figure 4. The amount of adsorption and the hydrodynamic layer thickness against the mole percent of DMAEM with a constant total degree of polymerization of ca. 200.

Figure 5. The amount of adsorption and the hydrodynamic layer thickness against the mole percent of DMAEM with a constant total degree of polymerization of ca. 700 . 
causes a decrease in the amount of adsorption. The increased DMAEM block length naturally decreases the BMA block length because of the constant total degree of polymerization. This decreased BMA block length leads to a decrease in the hydrodynamic layer thickness.

The experimental maximums occur at adsorbing block sizes smaller than those predicted by the theoretical model. Our results show the maximums occur at $4-5$ mole percents of the adsorbing block. According to the theory, when the bulk volume fraction of copolymer equals to $10^{-4}$, and $\chi$ parameters for surface and adsorbing block, surface and non-adsorbing block, adsorbing block and solvent, non-adsorbing block and solvent, and adsorbing and non-adsorbing block equal to - 8.0, 0.0, 0.5, 0.0 and 0.5 , respectively, the maximum occurs at 10 - 15 mole percents for the degree of polymerizations of 200 500. The theoretical calculations were made assuming monodispersity in molecular weight, while the experimental results were obtained with copolymers of narrow dispersity, but not monodisperse in molecular weight. The polydispersity of the adsorbing block is believed to be responsible for this difference in the location of the maximum. For copolymers with polydisperse adsorbing blocks, the adsorbed copolymers actually have larger adsorbing blocks than the average adsorbing block sizes of the original copolymers, which correspond to lower adsorbed amounts than those for the original copolymers. The maximums will tend to shift to the left of the curves.

These results are significant from practical point of view, in the design of diblock copolymers as colloid stabilizers. Polar adsorbing monomers are usually much more expensive than typical non-adsorbing blocks and at times have detrimental properties. Optimizing the adsorbing block size not only contributes to adsorption, but also cost and overall properties of the colloidal dispersions.

In the design of diblock copolymers as colloid stabilizers, it is also important to know how the layer thickness scales with the degree of polymerization of the non-adsorbing block. To prevent particles from aggregating, the layer thickness should be sufficiently large to shield the van der Waals attractive forces. A number of previous workers have proposed scaling relationships. Munch and Gast ${ }^{5}$ summarize the available measurement of block copolymer layer thickness in the light of these models. They suggest two regions for block copolymer adsorption: region I (adsorption dominated by packing of the adsorbing head groups for copolymers with large heads) and region II (adsorption dominated by the repulsion between the non-adsorbing blocks with small adsorbing heads). The scaling factors for regions I and II were found to be 1.0 and 0.7 , respectively. Our data on the amount of adsorption shows that the adsorbed amount solely due to the adsorbing group is always less than the adsorbed amount of the homopolymer of the adsorbing group. This indicates that our adsorption results are in region II. However, we found the scaling factor to be 0.53 as shown in Figure 6 (Note that we used data in the

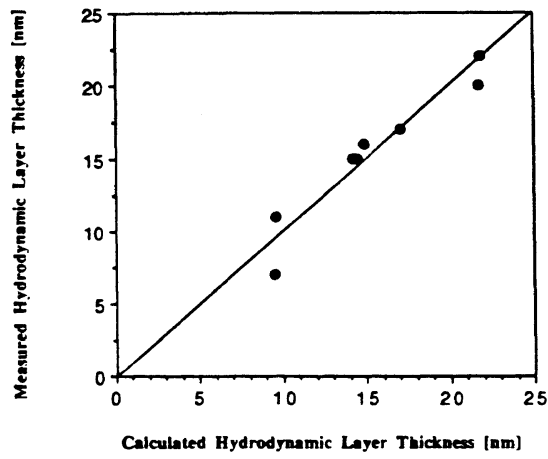

Figure 6. Comparison between the experimental data on the hydrodynamic layer thickness and the hydrodynamic layer thickness calculated from RA 0.0121 RB 0.527 . (RA: segment length of DMAEM; RB: segment length of BMA)

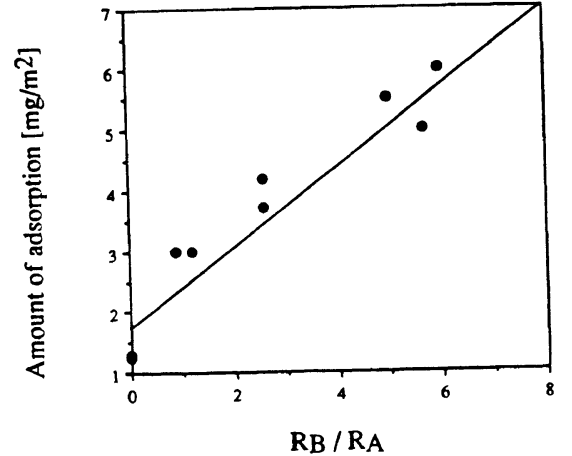

Figure 7. The adsorbed amount against $\mathrm{RA} / \mathrm{RB}$. 
right hand side of the maximums). Since the adsorbing blocks of our copolymers are soluble in 2-propanol whereas the layer thickness data used by Munch and Gast ${ }^{3}$ were obtained using diblock copolymers with insoluble adsorbing blocks, this difference may have contributed to the difference in the scaling factor.

Evers, et al. suggested a scaling relationship for the adsorbed amount for the mole percent of the adsorbing group greater than ca. $20 \%$ as follows:

$$
\theta_{\mathrm{T}}=\theta_{\mathrm{H}, \mathrm{A}}\left(1+\alpha \mathrm{R}_{\mathrm{B}} / \mathrm{R}_{\mathrm{A}}\right)
$$

where $\theta_{\mathrm{T}}$ is the total adsorbed amount of the copolymer, $\theta_{\mathrm{H}, \mathrm{A}}$ is the adsorbed amount for the homopolymer consisting of the adsorbing block, $\mathrm{A}$, of length equal to that of the copolymer (i. e., $R_{B}+R_{A}$ ), and $R_{B}$ and $R_{A}$ are the segment length of the non-adsorbing block, $\mathrm{B}$, and the adsorbing bock length, $\mathrm{A}$, respectively. Our experiment showed that $\theta_{\mathrm{H}, \mathrm{A}}$ is $1.3 \mathrm{mg} / \mathrm{m}^{2}$ and independent of the degree of polymerization (41 to 174). Thus, we could plot our data for different degrees of polymerization in the same figure (Figure 7). A good linearity was observed for the mole percent of the adsorbing group greater than $14 \%$ as the theory predicts. From the slope, $\alpha$ was found to be 0.42 .

\section{CONCLUSIONS}

1. With constant degrees of polymerization, both the adsorbed amount and the hydrodynamic layer thickness exhibit steep maximums as the adsorbing block composition is varied, in agreement with Evers' theory based on the self-consistent field theory.

2. The maximums in the adsorbed amount and the hydrodynamic layer thickness occur at adsorbing block sizes smaller than those predicted by the theory, probably due to the slight polydispersity of the copolymer.

3. The hydrodynamic layer thickness scales on the order of 0.53 with the degree of polymerization of non-adsorbing block, contrary to 0.7 found by other workers. This difference may be due to the soluble nature of the adsorbing block in our system.

4. A good agreement exits between our results and Evers' theory in terms of the linearity between the adsorbed amount and the ratio of the non-adsorbing block size to the adsorbing size.

\section{REFERENCES}

1. O. A. Evers, Ph D Thesis, Wageningen University, Netherlands (1990)

2. O. A. Evers, J. M. H. M. Scheutjens, and G. J. Fleer, J. Chem. Soc. Trans. Faraday Soc. , in press.

3. O. A. Evers, J. M. H. M. Scheutjens, and G. J. Fleer, Macromolecules, accepted for publication.

4. S. Patel, M. Tirrell, and G. Hadziioannou, Colloids Surf., 31, 157 (1988).

5. M. R. Munch, and A. P. Gast, Polymer Comm., 30, 324 (1989).

6. M. R. Stoeber, A. Fink, and E. Bohn, J. Colloid Interface Sci., 26, 62 (1968).

7. D. Y. Sogah, W. R. Hertler, O. W. Webster, and G. M. Cohen, Macromolecules, 20, 1473 (1987).

8. D. E. Koppel, J. Chem. Phys., 57, 4814 (1972).

9. J. C. Brown, P. N. Pusey, and R. Dietz, J. Chem. Phys., 62, 1136 (1975). 